\title{
AREA-MINIMIZING INTEGRAL CURRENTS WITH BOUNDARIES INVARIANT UNDER POLAR ACTIONS
}

\author{
JULIAN C. LANDER
}

\begin{abstract}
Let $G$ be a compact, connected subgroup of $S O(n)$ acting on $\mathbf{R}^{n}$, and let the action of $G$ be polar. (Polar actions include the adjoint action of a Lie group $H$ on the tangent space to the symmetric space $G / H$ at the identity coset.) Let $B$ be an $(m-1)$-dimensional submanifold without boundary, invariant under the action of $G$, and lying in the union of the principal orbits of $G$. It is shown that, if $S$ is an area-minimizing integral current with boundary $B$, then $S$ is invariant under the action of $G$. This result is extended to a larger class of boundaries, and to a class of parametric integrands including the area integrand.
\end{abstract}

1. Introduction. In this paper, we examine whether an area-minimizing surface inherits the symmetries of its boundary. We will prove the following theorem, which is the first result in general dimension and codimension.

THEOREM. Let $G$ be a compact, connected subgroup of $S O(n)$ acting on $\mathbf{R}^{n}$, and let the action of $G$ be polar. If $B$ is an $(m-1)$-dimensional submanifold without boundary, invariant under the action of $G$, and lying in the union of the principal orbits, then every area-minimizing surface (integral current) having $B$ as its boundary will also be invariant under the action of $G$.

A polar action allows a system of "polar coordinates" to be developed for $\mathbf{R}^{n}$. Polar actions are defined in 3.2. The action of $S O(k)$ on $\mathbf{R}^{n}$ is polar. For any symmetric space $G / H$, the adjoint action of $H$ on the tangent space to $G / H$ at $e H$ is polar.

This result also applies when area is replaced by a positive, even, elliptic, parametric integrand invariant under the group action which satisfies a symmetry condition defined in 4.3 .

There have been a number of previous results in this area. These apply exclusively to the codimension 1 problem, with the exception of the work of Bindschadler [1], which applies to surfaces of maximal codimension.

Lawson [8] proves that, given an $(n-2)$-dimensional submanifold of $\mathbf{S}^{n-1}$ without boundary, invariant under the action of a connected subgroup of $S O(n)$, there exists an area-minimizing integral current in $\mathbf{R}^{n}$ having this submanifold as its boundary, which is also invariant under the group action. Further, if there is only one such current, it is the unique area-minimizing current having that boundary.

Received by the editors May 5, 1986 and, in revised form, May 1, 1987. The contents of this paper were presented at a session of the 93rd Annual Meeting of the American Mathematical Society, January 21-24, 1987.

1980 Mathematics Subject Classification (1985 Revision). Primary 49F20, 49F22; Secondary $57 \mathrm{~S} 15,53 \mathrm{C} 35$.

This work was performed independently of the author's connection with Lincoln Laboratory. 
Brothers [2] gives a result for a group of orthogonal diffeomorphisms on a manifold whose orbits of highest dimension are connected. In this case, if there is an invariant integral current of codimension 1 , then every area-minimizing integral current with the same boundary will also be invariant under the group action.

Morgan [9, Theorem 7.4] gives a similar result for a connected group of diffeomorphisms on a complete, connected, noncompact, and orientable Riemannian manifold. He proves that every area-minimizing integral current having a boundary which is an invariant submanifold of codimension 2 will also be invariant.

Brothers and Morgan both extend their results to certain classes of parametric integrands.

Bindschadler [1] has proved our result for boundaries of maximal codimension. He requires that $G$ be a compact, connected subgroup of $S O(n)$ whose action is polar. He then proves that every area-minimizing integral current whose boundary consists of a finite number of principal $G$-orbits is invariant under the action. Bindschadler states his conditions for the group $G$ in a different form, not using the term polar. He states that the group should be compact and connected, and that the distribution of tangent planes orthogonal to the principal orbits should be involutive. This is equivalent to polarity, because the geodesics minimizing distance between the principal orbits lie in the integral manifolds of this distribution [4].

The idea behind the proof of our result is simple. The existence of polar coordinates for the group action allows the construction of slices of an arbitrary area-minimizing current with invariant boundary. The smallest (in some sense) of these is chosen, and its image under the group action is shown to have no more area than the original current. Complications arise because the theory of slicing fails on the nonprincipal orbits, so the slices must be approximations, and the compactness theorem for integral currents is used to find the desired current.

It is not known whether all the hypotheses of this result are necessary. The requirement that the group action be polar is very restrictive. While there are many known examples of area-minimizing integral currents not inheriting the symmetries of their boundaries (see $[5,5.4 .17 ; 2 ; 9, \S 8]$ ), each of these examples violates at least two of the hypotheses.

It may be possible to extend this result to the case of integral currents in a manifold. Palais and Terng [10] have extended the notion of a polar action to group actions on Riemannian manifolds. At this writing, there are no analogues of Dadok's results [3], which limit the sizes of the principal orbits of a group action near the singular orbits. These limits are necessary to the proof of the result of this paper.

$\S 2$ establishes the system of notation to be used throughout this paper. In $\S 3$, a theory of polar coordinates is developed, which relies heavily on the theory of symmetric spaces. The main result is developed in $\S 4$, and is stated and proved in 4.12 .

The results presented here are based on [7]. The author has taken the opportunity to simplify the proofs and corrrect some errors.

The author would like to express his thanks to Frank Morgan for his advice in developing and preparing this paper.

2. Notation. The notations and concepts used in this paper are taken from the treatise of Federer [5]. 
In addition, if $f: X \rightarrow Y$ is a function between normed vector spaces, $\|f\|_{\infty}$ denotes $\sup \{|f(x)|: x \in X\}$.

Some additional notations are established and used in $\S 3$ only. They relate to the theory of Lie groups and Lie algebras, and are defined in the introduction to $\S 3$.

3. Polar actions and coordinates. In this section, which is chiefly expository, we define polar actions and develop a system of polar coordinates for the spaces upon which they act. In 3.1-3.5, we examine polar actions and give their relation to symmetric space actions, relying on the work of Dadok [3]. In 3.6 to 3.9, the polar coordinates are developed. Many of the results here are from the treatise of Helgason [6], especially Chapter 7 . We conclude by defining some basic functions, which will be used extensively in the following section, in 3.10 to 3.12 .

3.1. Polar actions. Let $G$ be a connected, compact subgroup of $S O(n)$ acting on $\mathbf{R}^{n}$, and let $\mathfrak{g}$ denote its Lie algebra. We denote the inner product on $\mathbf{R}^{n}$ by $\langle\cdot, \cdot\rangle$ and the commutator operation on $\mathfrak{g}$ by $[\cdot, \cdot]$.

For $x \in \mathbf{R}^{n}$, let $\mathfrak{a}_{x}=\left\{y \in \mathbf{R}^{n}:\langle y, \mathfrak{g} \cdot x\rangle=0\right\}$ denote the orthogonal crosssection to the orbit of $G$ through $x$. The cross section $\mathfrak{a}_{x}$ intersects every $G$-orbit [3, Proposition 1]. We call the point $x$ regular if $G(x)$ has maximal dimension.

Proposition [3, Proposition 2]. Fix $x_{0} \in \mathbf{R}^{n}$, a regular point. The following are equivalent.

1. For any regular $x \in \mathbf{R}^{n}, \mathfrak{g} \cdot x=\sigma\left(\mathfrak{g} \cdot x_{0}\right)$ for some $\sigma \in G$.

2. For any regular $x \in \mathbf{R}^{n}, \mathfrak{a}_{x}=\sigma\left(\mathfrak{a}_{x_{0}}\right)$ for some $\sigma \in G$.

3. For any $x \in \mathfrak{a}_{x_{0}},\left\langle\mathfrak{g} \cdot x, \mathfrak{a}_{x_{0}}\right\rangle=0$.

3.2. Definition. An action of a connected, compact subgroup of $S O(n)$ on $\mathbf{R}^{n}$ having the properties described in 3.2 is called a polar action. A maximal orthogonal cross section $\mathfrak{a}_{x}$ for a polar action is called a Cartan subspace.

3.3. Definition. The action of a connected, compact subgroup of $G$ of $S O(V)$ with Lie algebra $\mathfrak{g}$ on a real vector space $V$ is a symmetric space action if there is a real semisimple Lie algebra $\mathfrak{u}$ with Cartan decomposition $\mathfrak{u}=\mathfrak{k}+\mathfrak{p}$, a Lie algebra isomorphism $A: \mathfrak{g} \rightarrow \mathfrak{k}$, and a real vector space isomorphism $L: V \rightarrow \mathfrak{p}$ such that $L(X \cdot y)=[A(X), L(y)]$ for all $X \in \mathfrak{g}$ and $y \in V$.

3.4. REMARK. If the action of $G$ is a symmetric space action, $U$ is a connected Lie group with Lie algebra $\mathfrak{u}$, and $K$ is a connected subgroup of $U$ with Lie algebra $\mathfrak{k}$, then the action of $G$ on $V$ is isomorphic to that of $\operatorname{Ad}(K)$ on $\mathfrak{p}$.

3.5. Polar and symmetric space actions. The relation between polar and symmetric space actions is provided by the following proposition.

PROPOSITION [3, PROPOSITION 6]. Let $G$ be a connected, compact subgroup of $S O(n)$ whose action on $\mathbf{R}^{n}$ is polar. Then there exists a connected subgroup $G^{\prime}$ of $S O(n)$ whose action on $\mathbf{R}^{n}$ is a symmetric space action and whose orbits coincide with those of $G$.

3.6. Roots. Let $\mathfrak{a}$ be a maximal abelian subspace of $\mathfrak{p}$, and let $\mathfrak{h}$ be a maximal abelian subspace of $\mathfrak{u}$ containing $\mathfrak{a}$. The roots of $\mathfrak{u}$ with respect to $\mathfrak{a}$ are the nonzero linear functionals $\lambda$ on a such that, for each $H \in \mathfrak{a}, \lambda(H)$ is an eigenvalue of $\operatorname{ad} H: \mathfrak{u} \rightarrow \mathfrak{u}$. Let $\Sigma$ denote the set of roots of $\mathfrak{u}$ with respect to $\mathfrak{a}$. For each $\lambda \in \Sigma$, let $\mathfrak{u}_{\lambda}$ denote the eigenspace of $\lambda$ in $\mathfrak{u}$, and let $m_{\lambda}=\operatorname{dim} \mathfrak{u}_{\lambda}$. Order the dual of $\mathfrak{a}$, 
and let $\Sigma^{+}$denote the set of positive roots with respect to this ordering. (For a more complete discussion, see [6, pp. 263-264]. What we have defined here as roots are there referred to as restricted roots.)

A root $\lambda \in \Sigma^{+}$is simple if it cannot be written as the sum of positive roots. Let $\left\{\lambda_{1}, \lambda_{2}, \ldots, \lambda_{l}\right\}$ be the set of simple roots in $\Sigma^{+}$. We have $l=\operatorname{dim} \mathfrak{a}$ [6, VII.2.19].

3.7. Polar coordinates. Let $C^{+}=\left\{H \in \mathfrak{a}: \lambda>0\right.$ for all $\left.\lambda \in \Sigma^{+}\right\}$. Let $X$ be the closure of $C^{+}$in a. $X$ contains exactly one point of each $\operatorname{Ad}(K)$-orbit in $\mathfrak{p}[\mathbf{6}$, VII.8.6]. Let $M$ denote the centralizer of $\mathfrak{a}$ in $K$, and let $\mathfrak{m}$ denote its Lie algebra.

Define the map $\phi: K / M \times X \rightarrow \mathfrak{p}$ by $\phi(\sigma M, H)=\operatorname{Ad}(\sigma) H$. The function $\phi$ defines the set of polar coordinates for $\mathfrak{p}$, allowing each point in $\mathfrak{p}$ to be expressed as an orbit and a coset of the isotropy subgroup. The function $\phi$ is clearly well defined, differentiable, and surjective.

We now examine $D \phi$. For $\sigma \in K$, let $\rho(\sigma): K \rightarrow K$ be defined by $\rho\left(\sigma_{1}\right) \sigma_{2}=$ $\sigma_{1} \sigma_{2}$. Let $\mathfrak{l}$ denote the orthogonal complement of $\mathfrak{m}$ in $\mathfrak{k}$. Since $\mathfrak{l}$ is isomorphic to the tangent space to $K / M$ at $e M, D \rho(\sigma) \mathfrak{l}$ may be identified with the tangent space to $K / M$ at $\sigma M$. Let $\left(\sigma_{0} M, H_{0}\right) \in K / M \times X, L \in \mathfrak{l}$, and $H \in \mathfrak{a}$. Then

$$
D \phi\left(\sigma_{0} M, H_{0}\right)\left(D \rho\left(\sigma_{0}\right) L, H\right)=\operatorname{Ad}\left(\sigma_{0}\right)\left(\left[L, H_{0}\right]+H\right)
$$

[6, pp. 294-295].

Note that, if $H_{0} \in C^{+}$, then $D \phi\left(\sigma_{0} M, H_{0}\right)$ is invertible, so $K / M \times C^{+}$is a local diffeomorphism.

3.8. REMARK. The action of $\operatorname{Ad}(K)$ on $\mathfrak{p}$ is polar, with $\mathfrak{a}$ the Cartan subspace $\mathfrak{a}_{H}$ for any $H \in C^{+}$.

3.9. The proof of the following proposition is standard. It may be found in the proof of [6, VII.3.1].

PROPOSITION. The restriction $\phi \mid K / M \times C^{+}$is a diffeomorphism onto its image, which consists precisely of the regular points of $\mathfrak{p}$ under the action of $\operatorname{Ad}(K)$.

3.10. Let $k=\operatorname{dim}(K / M)$, which is also the dimension of every regular $\operatorname{Ad}(K)$ orbit. We construct a function $\gamma: X \rightarrow \mathbf{R}$ which gives the $\mathcal{H}^{k}$ measure of every $\operatorname{Ad}(K)$-orbit. Define $\gamma$ by

$$
\gamma(H)=\prod_{\lambda \in \Sigma^{+}} \lambda(H)^{m_{\lambda}} .
$$

Proposition. For all $H \in X, \mathcal{H}^{k}(\operatorname{Ad}(K) H)=\gamma(H) \not^{k}(K / M)$.

PrOOF. Let $\theta$ be a unit $k$-vectorfield on $K / M$. By [6, VI.3.6], we have

$$
\theta(\sigma M)=\bigwedge_{k} D \rho(\sigma) \bigwedge_{k} \mathfrak{l}=\bigwedge_{\lambda \in \Sigma^{+}} \bigwedge_{i=1}^{m_{\lambda}} D \rho(\sigma)\left(X_{\lambda, i}+\tau X_{\lambda, i}\right),
$$

where $\left\{X_{\lambda, i} \in \mathfrak{u}_{\lambda}: i=1,2, \ldots, m_{\lambda}\right\}$ is an orthonormal basis for $\mathfrak{u}_{\lambda}$, and $\tau$ denotes conjugation in $\mathfrak{u}$ with respect to $\mathfrak{k}$.

Then we have

$$
\begin{aligned}
\mathscr{H}^{k}[\operatorname{Ad}(K) H] & =\nvdash^{k}[\phi(K / M, H)] \\
& =\int_{K / M}\left|\bigwedge_{k} D \phi(\sigma M, H) \theta(\sigma M)\right| d \nvdash^{k}(\sigma M) \\
& =\mathcal{H}^{k}(K / M)\left|\bigwedge_{k} D \phi(e M, H) \theta(e M)\right|
\end{aligned}
$$


the first by [5, 3.2.22], and the second because the action of $\operatorname{Ad}(K)$ is orthogonal.

Note that

$$
\bigwedge_{k} D \phi(e M, H) \theta(e M)=\bigwedge_{\lambda \in \Sigma^{+}} \bigwedge_{i=1}^{m_{\lambda}} \lambda(H)\left(X_{\lambda, i}-\tau X_{\lambda, i}\right)
$$

and

$$
\left|X_{\lambda, i}+\tau X_{\lambda, i}\right|=\left|X_{\lambda, i}-\tau X_{\lambda, i}\right|
$$

completing the proof of the proposition.

3.11. Define the function $d: X \rightarrow \mathbf{R}$ by

$$
d(H)=\inf \left\{\lambda_{i}(H): i=1,2, \ldots, l\right\}
$$

where the $\lambda_{i}$ are the simple roots in $\Sigma^{+}$. The value of $d(H)$ is, in some sense, the distance from $H$ to the boundary of $X$. It is clear that the function $d$ is Lipschitzian.

3.12. Choose a dual basis to the $\lambda_{i}$, a set $\left\{\xi_{i} \in \mathfrak{a}: \lambda_{i}\left(\xi_{j}\right)=\delta_{j}^{i}\right.$ for $i, j=$ $1,2, \ldots, l\}$, where $\delta$ is the Kronecker delta function. Define the function $h: C^{+} \rightarrow$ $C^{+}$by $h(H)=d(H) \sum_{i=1}^{l} \xi_{i} / \lambda_{i}(H)$. This function has the important property that $d[H-d(H) h(H)]=0$. It is locally Lipschitzian and $\|h\|_{\infty}$ is finite.

PROPOSITION. There exists a constant $C_{h}$ such that, if $h$ is approximately differentiable at $H \in C^{+}$, then $\|\operatorname{ap} D h(H)\| \leq C_{h} / d(H)$.

Proof. For $H \in C^{+}$, define $g(H)=\sum_{i=1}^{l} \xi_{i} / \lambda_{i}(H)$. Then

$$
|g(H)| \leq \sum_{i=1}^{l}\left|\xi_{i}\right| / d(H),
$$

and $g$ is differentiable everywhere in $C^{+}$with

$$
\|D g(H)\| \leq \sum_{i=1}^{l}\left|\xi_{i}\right| \sup \left\{\left\|\lambda_{i}\right\|\right\} / d^{2}(H) .
$$

Then, since $h(H)=d(H) g(H)$,

$$
\|\operatorname{ap} D h(H)\| \leq d(H)\|D g(H)\|+\operatorname{Lip}(d)|g(H)|,
$$

completing the proof with

$$
C_{h}=\sum_{i=1}^{l}\left|\xi_{i}\right| \sup \left\{\left\|\lambda_{i}\right\|\right\}+\operatorname{Lip}(d) \sum_{i=1}^{l}\left|\xi_{i}\right| .
$$

4. Integral currents with invariant boundaries. In this section, we prove the main result of this paper.

Let $G$ be a connected, compact subgroup of $S O(n)$ whose action on $\mathbf{R}^{n}$ is polar. Let $k$ be the dimension of the principal orbits of $G$, and let $Z$ denote the union of the principal orbits.

Let $m$ be a positive integer, with $k<m \leq n$. Let $B$ be an $(m-1)$-dimensional integral current in $\mathbf{R}^{n}$ invariant under the action of $G$ and without boundary. Further, assume that $B=B\left\lfloor Z\right.$ and that $\mathcal{H}^{m}[\operatorname{spt}(B)]=0$. (The last two conditions are satisfied if $B$ is a compact, invariant submanifold of $Z$.) 
Let $S$ be an $m$-dimensional integral current lying in $\mathbf{R}^{n}$ with boundary $B$. We will prove that $S$ is an area-minimizing integral current with boundary $B$ only if $S$ is invariant under the action of $G$. This result will be extended to the case in which area is replaced by a member of a more general class of integrands defined in 4.3.

In 4.1, we will construct a system of polar coordinates for the action of $G$, and in 4.2 we establish some preliminary concepts and notation. The class of integrands with which we are concerned is defined in 4.3.

Our proof will consist of constructing an integral current $T$ with boundary $B$ whose area is no greater than that of $S$. In 4.4 and 4.5 , we show that the boundary of $T$ is $B$. In 4.6, we construct $T$ for the case in which $\operatorname{spt}(B) \subset Z$. This is extended to the general case in 4.7-4.9. In 4.10 and 4.11, we show that $S$ and $T$ have equal area only if $S$ is invariant under the action of $G$. The main result is proved in 4.12.

4.1. Polar coordinates for the group action. Since $G$ is a connected, compact group whose action on $\mathbf{R}^{n}$ is polar, the results of $\S 3$ apply. By 3.5 , we may assume that the action of $G$ is a symmetric space action, and that $G=\operatorname{Ad}(K)$ for the Lie group $K$ described in 3.6, identifying $\mathbf{R}^{n}$ with $\mathfrak{p}$. Let $H=\operatorname{Ad}(M)$, where $M$ is the isotropy subgroup defined in 3.9.

Using the identifications above, and noting that $G / H$ is isomorphic to $K / M$, we can use the functions defined in $\S 3$ for the action of $G$ on $\mathbf{R}^{n}$.

The function $\phi$ becomes a function $\phi: G / H \times X \rightarrow \mathbf{R}^{n}$. Define $f: Z \rightarrow G / H^{*}$ and $p: Z \rightarrow X$ by $\phi^{-1} \mid Z=f \times p$. The function $p$ can be extended to all of $\mathbf{R}^{n}$, since $X$ contains exactly one point from each $G$-orbit.

The functions $\gamma: X \rightarrow \mathbf{R}$, defined in $3.10 ; d: X \rightarrow \mathbf{R}$, defined in 3.11 ; and $h: \operatorname{Int}(X) \rightarrow \operatorname{Int}(X)$, defined in 3.12 , will also be used in this section.

4.2. Preliminaries. Let $\theta$ be a unit orienting $k$-vectorfield on $G / H$, and let $\theta^{*}$ be its dual $k$-form. Let $\vec{Y}$ be a unit orienting $k$-vectorfield on $Z$ associated with the $k$-plane tangent to the orbits of $G$, so that

$$
\left\langle\bigwedge_{k} D \phi(g H, x), \theta(g H)\right\rangle=\gamma(x) \vec{Y}[\phi(g H, x)] .
$$

Let $Y^{*}$ be the $k$-form dual to $\vec{Y}$.

For each $z \in Z$, let $P_{z}$ be the $(n-k)$-plane orthogonal to the action of $G$ at $z$. If $P_{0}$ is the $(n-k)$-plane containing $X$, then $P_{z}=\phi\left[f(z), P_{0}\right]$.

For each $\delta>0$, define the sets $D_{\delta}=\left\{z \in \mathbf{R}^{n}: d(z)>\delta\right\}$ and $C_{\delta}=\{z \in$ $\left.\mathbf{R}^{n}: d(z) \leq \delta\right\}$

Choose $P_{S} \in \mathbf{R}^{+}$such that $\operatorname{spt}(S) \subset \mathbf{B}\left(0, P_{S}\right)$, where $\mathbf{B}\left(0, P_{S}\right)$ denotes the closed ball of radius $P_{S}$ about the origin in $\mathbf{R}^{n}$.

4.3. Symmetric integrands. Let $\Psi$ be a positive, even, semielliptic parametric integrand of degree $m$ on $\mathbf{R}^{n}$. We call $\Psi$ invariant under the action of $G$ if, for all $g \in G, g^{\#} \Psi=\Psi$.

Assume that $z \in Z$ is fixed. Let $\xi \in \bigwedge_{m}\left(\mathbf{R}^{n}\right)$ with $\xi=\varsigma \wedge \vec{Y}(z)+\eta$ and $\eta\left\llcorner Y^{*}(z)=0\right.$. We call $\Psi$ symmetric if, for all $z \in Z, \Psi[z, \varsigma \wedge \vec{Y}(z)] \leq \Psi(z, \xi)$, with equality if and only if $\eta=0$.

If $\Psi$ is an integrand symmetric and invariant under the action of $G$, we define the $(m-k)$-dimensional integrand $\Psi^{*}$ on $X$ by

$$
\Psi^{*}(x, \varsigma)=\Psi\left\{\phi(e H, x), \vec{Y}[\phi(e H, x)] \wedge\left\langle\bigwedge_{m-k} D \phi(e H, x), \varsigma\right\rangle\right\}
$$

for $x \in X$ and $\varsigma \in \bigwedge_{m-k}\left(P_{0}\right)$. 
4.4. LEMMA. Let $E$ be an $\mathcal{H}^{j}$ measurable and $\left(\mathcal{H}^{j}, j\right)$ rectifiable subset of $Z$ such that $g(E)$ is $\mathcal{H}^{j}$ almost equal to $E$ for all $g \in G$. Then, for $\not^{j}$ almost all $z \in E, \operatorname{Tan}[G(z), z] \subset \operatorname{Tan}^{j}\left(\mathcal{H}^{j} L E, z\right)$.

PROOF. By $[5,3.2 .16]$, it suffices to show that, for every $\nu \in \operatorname{Tan}[G(z), z]$, $|\nu|=1$,

$$
\Theta^{j}\left\{\not^{j} L[E \cap \mathbf{E}(z, \nu, \varepsilon)]\right\}>0
$$

for every $\varepsilon>0$. Note that $\mathbf{E}(z, \nu, \varepsilon)=\bigcup_{t>0} \mathbf{U}(z+t \nu, t \varepsilon)$.

Fix $\varepsilon>0$. Since $\nu \in \operatorname{Tan}[G(z), z]$, there exists a one-parameter subgroup $\sigma(t)$ of $G$ such that, for all $t$ with $0 \leq t \leq t_{0},|\sigma(t) \cdot z-z-t \nu|<t \varepsilon / 2$. Hence, $\mathbf{U}[\sigma(t) \cdot z, t \varepsilon / 2] \subset \mathbf{E}(z, \nu, \varepsilon)$ and $\mathbf{U}[\sigma(t) \cdot z, t \varepsilon / 2] \subset \mathbf{U}[z, t(1+\varepsilon) / 2]$.

Using the definition of density $[5,2.10 .9]$, the invariance of $E$ under the action of $G$, and $[5,3.2 .19]$, we have

$$
\begin{aligned}
\Theta^{j}\left\{\not^{j} L[E \cap \mathbf{E}(z, \nu, \varepsilon)]\right\} & \geq \lim _{t \rightarrow \infty} \alpha(j)^{-1}[t(1+\varepsilon) / 2]^{-j}\left(\not \not^{j} L E\right) \mathbf{U}[\sigma(t) \cdot z, t \varepsilon / 2] \\
& \geq[\varepsilon /(2+\varepsilon)]^{j} \Theta^{j}\left(\not^{j} L E, z\right) \geq[\varepsilon /(2+\varepsilon)]^{j},
\end{aligned}
$$

completing the proof of the lemma.

4.5. We will use this lemma to prove the following theorem, which will show that the new current $T$ has boundary $B$.

THEOREM. Let $B \in \mathbf{I}_{m-1}(Z)$ be invariant under the action of $G$. Then, for all $g H \in G / H, B=\phi_{\#}\left(G / H \times p_{\#}\langle B, f, g H\rangle\right)$.

Proof. Fix $g_{0} \in G$, and let $B^{\prime}=\phi_{\#}\left(G / H \times p_{\#}\left\langle B, f, g_{0} H\right\rangle\right)$. We will show that, for any $\beta \in D^{m-1}(Z), B^{\prime}(\beta)=B(\beta)$.

Let $\beta \in D^{m-1}(Z)$. Define $\beta_{G} \in D^{m-1}(Z)$ by $\beta_{G}=\int_{G} g^{\#} \beta d g$, using Haar measure on $G$, normalized so that $\int_{G} d g=1$.

Since $B$ and $B^{\prime}$ are both invariant under the action of $G, B(\beta)=B\left(\beta_{G}\right)$ and $B^{\prime}(\beta)=B^{\prime}\left(\beta_{G}\right)$. Using these identities and the definition of $B^{\prime}$ above, and expanding the functions, we have

$$
B^{\prime}(\beta)=(-1)^{k(m-k-1)} \not^{k}(G / H)\langle B, f, g H\rangle\left[\gamma \wedge\left(\vec{Y}\left\llcorner\beta_{G}\right)\right] .\right.
$$

By the invariance of $B$ and $[5,4.3 .2(7)]$, we see that $\left\langle B, f, g_{0} H\right\rangle=\langle B, f, g H\rangle$ for all $g \in G$. Finally, we apply $[\mathbf{5}, 4.3 .2(1)]$ to obtain

$$
\begin{aligned}
B^{\prime}(\beta) & =(-1)^{k(m-k-1)} \int_{G / H}\langle B, f, g H\rangle\left[\gamma \wedge\left(\vec{Y}\left\llcorner\beta_{G}\right)\right] d \not^{k}(g H)\right. \\
& =B(\beta),
\end{aligned}
$$

completing the proof.

4.6. THEOREM. Let $S \in \mathbf{I}_{m}\left(\mathbf{R}^{n}\right)$ with $B=\partial S$ invariant under the action of $G$ and $\operatorname{spt}(B) \subset Z$. Then there exists $T \in \mathbf{I}_{m}\left(\mathbf{R}^{n}\right)$ such that

1. $\partial T=B$,

2. $T$ is invariant under the action of $G$, and

3. $\Psi(T) \leq \Psi\left\{\left[S\left\llcorner\left(Z \wedge Y^{*}\right)\right] \wedge \vec{Y}\right\} \leq \Psi(S)\right.$.

PrOOF. The current $T$ is essentially $\phi_{\#}\left(G / H \times p_{\#}\langle S L Z, f, g H\rangle\right)$ for a suitable $g H \in G / H$. We first show that an appropriate choice of $g H$ exists, and then consider the problem of introducing extra boundary. 
Before we begin, we note that, if $W \in R_{m}\left(\mathbf{R}^{n}\right)$ and $U$ is an open subset of $\mathbf{R}^{n}$, then $W L U \in R_{m}\left(\mathbf{R}^{n}\right)$.

We begin by using $[\mathbf{5}, 4.3 .2(2)]$ and $[\mathbf{5}, 4.3 .8(2)]$ to see that

$$
\int_{G / H} \Psi\left[\left(\langle S\llcorner Z, f, g H\rangle \wedge \vec{Y})\llcorner\gamma] d \not^{k} g H \leq \Psi\left\{\left[S\left\llcorner\left(Z \wedge Y^{*}\right)\right] \wedge \vec{Y}\right\} .\right.\right.\right.
$$

Thus, there exists $\Gamma \subset G / H$ with $\nvdash^{k}(\Gamma)>0$ such that, for all $g H \in \Gamma$,

$$
\Psi^{*}\left(p _ { \# } \left\langleS\llcorner Z, f, g H\rangle\llcorner\gamma) \leq \Psi\left\{\left[S\left\llcorner\left(Z \wedge Y^{*}\right)\right] \wedge \vec{Y}\right\} / \not^{k}(G / H)\right. \text {. }\right.\right.
$$

Further, by $[\mathbf{5}, 4.3 .6]$ we may assume that $p_{\#}\left\langle S\llcorner Z, f, g H\rangle \in \mathbf{I}_{m-k}(X)\right.$ for all $g H \in \Gamma$.

We now consider the boundary problem. Let $\delta_{0}=\sup \left\{\rho \in \mathbf{R}: \operatorname{spt}(B) \subset D_{\rho}\right\}$. For every $\rho$ such that $0<\rho<\delta_{0}$,

$$
\partial\left(p_{\#}\left\langle S\left\llcorner D_{\rho}, f, g H\right\rangle\right)=(-1)^{k}\langle B, f, g H\rangle+(-1)^{k+1}\langle\langle S, d, \rho\rangle, f, g H\rangle .\right.
$$

We need an upper bound on the mass of the second term. Let $\delta>0$ be such that $2 \delta<\delta_{0}$. Applying [5, 4.3.2(2)], we have

$$
\begin{aligned}
\int_{\delta}^{2 \delta} & \int_{\Gamma} \mathbf{M}\left(\langle\langle S, d, \rho\rangle, f, g H\rangle\llcorner\gamma) d \not^{k}(g H) d \mathcal{L}^{1} \rho\right. \\
& =\int_{\delta}^{2 \delta} \mathbf{M}\left[\left(\langle S, d, \rho\rangle\llcorner\gamma) f^{\#}\left(\Gamma \wedge \theta^{*}\right)\right] d \mathcal{L}^{1} \rho\right. \\
& \leq \int_{\delta}^{2 \delta} \mathbf{M}\left(\langle S, d, \rho\rangle\left\llcorner Y^{*}\right) d \mathcal{L}^{1} \rho\right. \\
& \leq \operatorname{Lip}(d) \mathbf{M}\left\{S\left\llcorner\left[\left(D_{\delta} \cap C_{2 \delta}\right) \wedge Y^{*}\right]\right\} .\right.
\end{aligned}
$$

Thus, we can choose $\rho \in(\delta, 2 \delta)$ and $g H \in \Gamma$ such that

$$
\mathbf{M}\left(\langle\langle S, d, \rho\rangle, f, g H\rangle\llcorner\gamma) \leq \operatorname{Lip}(d) \mathbf{M}\left\{S\left\llcorner\left[\left(D_{\delta} \cap C_{2 \delta}\right) \wedge Y^{*}\right]\right\} / \delta \mathcal{H}^{k}(\Gamma) .\right.\right.
$$

Define $\mu:[0, \rho] \times X \rightarrow X$ by $\mu(t, x)=x-\operatorname{th}(x)$. Let

$$
W=-\mu_{\#}([0, \rho] \times\langle\langle S, d, \rho\rangle, f, g H\rangle) .
$$

Then $\operatorname{spt}(W) \subset \mathbf{B}\left(0, P_{S}+\delta_{0}\|h\|_{\infty}\right)$, and $\partial W=\langle\langle S, d, \rho\rangle, f, g H\rangle$. Note that $\operatorname{spt}(\langle\langle S, d, \rho\rangle, f, g H\rangle) \subset d^{-1}\{\rho\}$ and $\gamma \circ \mu \leq \gamma$.

Then, by $[5,4.1 .30]$, we have

$$
\begin{aligned}
\mathbf{M}(W\llcorner\gamma) & \leq \rho\|h\|_{\infty}\left(1+C_{h}\right)^{m-k-1} \mathbf{M}(\langle\langle S, d, \rho\rangle, f, g H\rangle\llcorner\gamma) \\
& \leq \operatorname{Lip}(d)\|h\|_{\infty}\left(1+C_{h}\right)^{m-k-1} \mathbf{M}\left\{S\left\llcorner\left[\left(D_{\delta} \cap C_{2 \delta}\right) \wedge Y^{*}\right]\right\} / \not^{k}(\Gamma) .\right.
\end{aligned}
$$

Let

$$
T_{\delta}=\phi_{\#}\left\{G / H \times\left[(-1)^{k} p_{\#}\left\langle S\left\llcorner D_{\rho}, f, g H\right\rangle+W\right]\right\} .\right.
$$

It is clear that $T_{\delta}$ is invariant under the action of $G$ and that $\partial T_{\delta}=B$. By $[\mathbf{5}$, 4.1.30],

$$
\begin{aligned}
\Psi\left(T_{\delta}\right) & \leq\left[\Psi ^ { * } \left(p _ { \# } \left\langleS\left\llcorner D_{\rho}, f, g H\right\rangle\llcorner\gamma)+\Psi^{*}(W\llcorner\gamma)] \mathcal{H}^{k}(G / H)\right.\right.\right. \\
& \leq \Psi\left\{\left[S\left\llcorner\left(Z \wedge Y^{*}\right)\right] \wedge \vec{Y}\right\}+\Psi^{*}\left(W\llcorner\gamma) H^{k}(G / H) .\right.\right.
\end{aligned}
$$

Since $\mathbf{B}\left(0, P_{S}+\delta_{0}\|h\|_{\infty}\right)$ is compact, there exists a constant $c$ such that

$$
\Psi^{*}(W\llcorner\gamma) \leq c \mathbf{M}(W \mathrm{~L} \gamma)
$$


Thus,

$$
\begin{aligned}
& \Psi\left(T_{\delta}\right) \leq \Psi\left\{\left[S\left\llcorner\left(Z \wedge Y^{*}\right)\right] \wedge \vec{Y}\right\}\right. \\
& \quad+c \operatorname{Lip}(d)\|h\|_{\infty}\left(1+C_{h}\right)^{m-k-1} \not^{k}(G / H) \mathbf{M}\left\{S\left\llcorner\left[\left(D_{\delta} \cap C_{2 \delta}\right) \wedge Y^{*}\right]\right\} / \not^{k}(\Gamma) .\right.
\end{aligned}
$$

Consider a sequence $\{\delta\}$ approaching zero. By the compactness theorem for integral currents $[\mathbf{5}, 4.2 .17]$, there is a convergent subsequence of the $T_{\delta}$. Let $T$ be the limit of this subsequence. Since $\lim _{\delta \rightarrow 0} \mathbf{M}\left\{S\left\llcorner\left[\left(D_{\delta} \cap C_{2 \delta}\right) \wedge Y^{*}\right]\right\}=0, T\right.$ will fulfill the conditions of this theorem.

4.7. We now extend Theorem 4.6 to the case in which the support of the boundary $B$ intersects the nonprincipal orbits. We still require that $B=B L Z$. The proof is accomplished by approximating an arbitrary current $S$ with boundary $B$ by a series of currents $S_{\delta}$ with boundaries $B_{\delta}$, to which Theorem 4.6 will apply.

For each $\delta>0$, define $\mu_{\delta}: \mathbf{R}^{n} \rightarrow \mathbf{R}^{n}$ by

$$
\mu_{\delta}(z)=\left\{\begin{array}{l}
z, \quad \text { if } d(z) \leq \delta \\
\phi\{f(z), p(z)-[d(z)-\delta] h(z)\}, \quad \text { if } d(z) \geq \delta .
\end{array}\right.
$$

Note that $\mu_{\delta}$ is locally Lipschitzian, $d \circ \mu_{\delta} \leq \delta$, and $\gamma \circ \mu_{\delta}(z) \leq \gamma(z) \delta / d(z)$.

PROPOSITION. There exists a constant $C_{\mu}$ such that, if $\mu_{\delta}$ is approximately differentiable at $z$, then for all $\varsigma \in P_{z}$ with $|\zeta|=1,\left|\left\langle\operatorname{ap} D \mu_{\delta}, \varsigma\right\rangle\right| \leq C_{\mu}$.

ProOF. Let $\nu_{\delta}: P_{0} \rightarrow P_{0}$ be defined by

$$
\nu_{\delta}(x)=\left\{\begin{array}{l}
x, \quad \text { if } d(x) \leq \delta, \\
x-[d(x)-\delta] h(x), \quad \text { if } d(x) \geq \delta .
\end{array}\right.
$$

It suffices to show that, if $\nu_{\delta}$ is approximately differentiable at $x$, then $\left\|\operatorname{ap} D \nu_{\delta}(x)\right\|$ $\leq C_{\mu}$. Collecting previous results on $d$ and $h$, including 3.12 , we have

$$
\begin{aligned}
\left\|\operatorname{ap} D \nu_{\delta}(x)\right\| & =1+\operatorname{Lip}(d)|h(x)|+[d(x)-\delta] C_{h} / d(x) \\
& \leq 1+\operatorname{Lip}(d)\|h\|_{\infty}+C_{h},
\end{aligned}
$$

proving the proposition for

$$
C_{\mu}=1+\operatorname{Lip}(d)\|h\|_{\infty}+C_{h} .
$$

4.8. LEMMA. Let $R \in R_{j}\left(\mathbf{R}^{n}\right)$ be such that $R=\left[R L\left(Z \wedge Y^{*}\right)\right] \wedge \vec{Y}$. Then $\lim _{\delta \rightarrow 0} \mathbf{M}\left(R-\mu_{\delta \#} R\right)=0$.

ProOF. For each $\delta<1$,

$$
R=R\left\llcorner C_{\delta}+R\left\llcorner\left(D_{\delta} \cap C_{\sqrt{\delta}}\right)+R\left\llcorner D_{\sqrt{\delta}} .\right.\right.\right.
$$

Since $\mu_{\delta} \mid C_{\delta}$ is the identity, it is clear that $\lim _{\delta \rightarrow 0} \mathbf{M}\left[\mu_{\delta \#}\left(R L C_{\delta}\right)\right]=0$. By 4.7, $\left\|\operatorname{ap} J_{j} \mu_{\delta}(z)\right\| \leq C_{\mu}^{j-k} \delta / d(z)$. Then

$$
\mathbf{M}\left\{\mu_{\delta \#}\left[R\left\llcorner\left(D_{\delta} \cap C_{\sqrt{\delta}}\right)\right]\right\} \leq C_{\mu}^{j-k} \mathbf{M}\left[R\left\llcorner\left(D_{\delta} \cap C_{\sqrt{\delta}}\right)\right]\right.\right.
$$

whose limit as $\delta$ approaches 0 is 0 . Finally,

$$
\mathbf{M}\left[\mu_{\delta \#}\left(R\left\llcorner D_{\sqrt{\delta}}\right)\right] \leq C_{\mu}^{j-k \sqrt{\delta}} \mathbf{M}\left(R\left\llcorner D_{\sqrt{\delta}}\right)\right.\right.
$$

which also approaches 0 as $\delta$ approaches 0 , completing the proof of the proposition. 
4.9. THEOREM. Let $S \in \mathbf{I}_{m}\left(\mathbf{R}^{n}\right)$ with $B=\partial S$ invariant under the action of $G$ and $B=B\left\llcorner Z\right.$. Then there exists $T \in \mathbf{I}_{m}\left(\mathbf{R}^{n}\right)$ such that

1. $\partial T=B$,

2. $T$ is invariant under the action of $G$, and

3. $\Psi(T) \leq \Psi\left\{\left[S\left\llcorner\left(Z \wedge Y^{*}\right)\right] \wedge \vec{Y}\right\} \leq \Psi(S)\right.$.

ProOF. Choose a sequence $\{\delta\}$ approaching 0 . For each $\delta$, let $S_{\delta}=S-\mu_{\delta \#} S$ and $B_{\delta}=B-\mu_{\delta \#} B$. Note that $\partial S_{\delta}=B_{\delta}$, and $\operatorname{spt}\left(B_{\delta}\right) \subset d^{-1}[\delta, \infty) \subset Z$. Applying 4.6, we have, for each $\delta, T_{\delta} \in \mathbf{I}_{m}\left(\mathbf{R}^{n}\right)$ such that

1. $\partial T_{\delta}=B_{\delta}$,

2. $T_{\delta}$ is invariant under the action of $G$, and

3. $\Psi\left(T_{\delta}\right) \leq \Psi\left\{\left[S_{\delta} L\left(Z \wedge Y^{*}\right)\right] \wedge \vec{Y}\right\} \leq \Psi\left(S_{\delta}\right)$.

By the compactness theorem for integral currents $[5,4.2 .17]$, there exists a convergent subsequence of the $T_{\delta}$. Let $T$ be the limit of this subsequence. The boundary $B$ fulfills the hypotheses of 4.8 by 4.3 , so $\partial T=B$. It is clear that $T$ is invariant under the action of $G$.

For each $\delta,\left[S_{\delta} L\left(Z \wedge Y^{*}\right)\right] \wedge \vec{Y}$ also satisfies the hypotheses of 4.8. Thus,

$$
\left[S\left\llcorner\left(Z \wedge Y^{*}\right)\right] \wedge \vec{Y}=\lim _{\delta \rightarrow 0}\left[S_{\delta}\left\llcorner\left(Z \wedge Y^{*}\right)\right] \wedge \vec{Y},\right.\right.
$$

and $\Psi(T) \leq \Psi\left\{\left[S\left\llcorner\left(Z \wedge Y^{*}\right)\right] \wedge \vec{Y}\right\}\right.$, completing the proof of the theorem.

4.10. The following lemma is true for any Riemannian manifold $U$ and any compact, connected Riemannian manifold $G / H$.

LEMMA [1, 3.5]. Let $U$ be an open subset of $X$. If

$$
W \in \mathbf{F}_{m}^{\mathrm{loc}}(U \times G / H) \cap\left\{T \in D_{m}(U \times G / H): \mathbf{M}(T)<\infty\right\}
$$

is such that

1. for $\|W\|$ almost all $(x, g H) \in U \times G / H$ there exists $\xi \in \bigwedge_{m-k} P_{0}$ such that $W(x, g H)=\xi \wedge \theta(g H)$, and

2. $\partial W=0$,

then there exists $R \in \mathbf{F}_{m-k}^{\mathrm{loc}} \cap\left\{T \in D_{m-k}(U): \mathbf{M}(T)<\infty\right\}$ such that $W=R \times G / H$.

4.11. This lemma is based on $[1,4.2]$.

LEMMA. If $S \in \mathbf{I}_{m}\left(\mathbf{R}^{n}\right)$ is such that

1. $\Psi(S)=\Psi\left\{\left[S\left\llcorner\left(Z \wedge Y^{*}\right)\right] \wedge \vec{Y}\right\}\right.$, and

2. $\mathcal{H}^{m}[\operatorname{spt}(\partial S)]=0$,

then $S$ is invariant under the action of $G$.

ProOF. Let $\left\{V_{j}: j \in J\right\}$ be a locally finite open cover of $p[Z-\operatorname{spt}(\partial S)]$ such that $\phi^{-1}$ is Lipschitzian on $\phi\left(G / H \times V_{j}\right)$. We can shrink the $V_{j}$ to a locally finite open cover $\left\{U_{j}: j \in J\right\}$ of $p[Z-\operatorname{spt}(\partial S)]$ such that $\mathrm{Cl}\left(U_{j}\right) \subset V_{j}$ for each $j \in J[6,1.15 .3]$. Let $\left\{\mu_{j}: j \in J\right\}$ be a partition of unity subordinate to $\left\{\phi\left(G / H \times U_{j}\right): j \in J\right\}$.

Let $S_{j}$ denote the functional restriction of $S$ to $D^{m}\left[\phi\left(G / H \times U_{j}\right)\right]$. Then $S_{j} \in$ $\mathrm{N}_{m}^{\text {loc }}\left(G / H \times U_{j}\right)$ and $\partial\left(\phi^{-1} \# S_{j}\right)=0$. Further, since $\Psi(S)=\Psi\left\{\left[S\left\llcorner\left(Z \wedge Y^{*}\right)\right] \wedge \vec{Y}\right\}\right.$, for $\|S\|$ almost every $z \in \mathbf{R}^{n}$, there exists $\xi \in \bigwedge_{m-k} P_{z}$ such that $S(z)=\xi \wedge \vec{Y}(z)$. By 4.10, there exists $R \in \mathbf{F}_{m-k}^{\text {loc }}\left(U_{j}\right) \cap\left\{T \in D_{m-k}\left(U_{j}\right): \mathbf{M}(T)<\infty\right\}$ such that $S_{j}=\phi_{\#}(R \times G / H)$, so $S_{j}$ is invariant under the action of $G$. For each $j, S\left\llcorner\mu_{j}=\right.$ $S_{j}\left\llcorner\mu_{j}\right.$, so $S\left\llcorner\mu_{j}\right.$ is also invariant under the action of $G$. 
Note that $S L[Z-\operatorname{spt}(\partial S)]=\sum_{j \in J} S L \mu_{j}$, and that $S \mathrm{~L}[Z-\operatorname{spt}(\partial S)]=$ $S\left\llcorner\left[\mathbf{R}^{n}-\operatorname{spt}(\partial S)\right]\right.$, since

$$
\Psi(S)=\Psi\left\{\left[S \mathrm{~L}\left(Z \wedge Y^{*}\right)\right] \wedge \vec{Y}\right\} \leq \Psi(S \mathrm{~L} Z) \leq \Psi(S) .
$$

Finally, $\operatorname{spt}\left\{S-S \mathrm{~L}\left[\mathbf{R}^{n}-\operatorname{spt}(\partial S)\right]\right\} \subset \operatorname{spt}(\partial S)$, so, by $[\mathbf{5}, 4.1 .20], S=$ $S\left\llcorner\left[\mathbf{R}^{n}-\operatorname{spt}(\partial S)\right]\right.$, and is therefore invariant under the action of $G$.

4.12. We now state and prove the main result of this paper.

THEOREM. Let $G$ be a compact, connected subgroup of $S O(n)$ whose action on $\mathbf{R}^{n}$ is polar. Let $\Psi$ be a positive, even, semielliptic parametric integrand of degree $m$ on $\mathbf{R}^{n}$ which is invariant under the action of $G$ and satisfies the symmetry condition of 4.3. (The area integrand fulfills this condition.) Let $B$ be an $(m-1)$ dimensional integral current in $\mathbf{R}^{n}$ without boundary, invariant under the action of $G$, with $\mathfrak{H}^{m}[\operatorname{spt}(B)]=0$, and with $B=B\llcorner Z$, where $Z$ is the union of the principal orbits of $G$. If $S$ is a $\Psi$-minimizing m-dimensional integral current with boundary $B$, then $S$ is invariant under the action of $G$.

PrOOF. Because of the conditions imposed on $\Psi$,

$$
\Psi(S) \geq \Psi\left\{\left[S \mathrm{~L}\left(Z \wedge Y^{*}\right)\right] \wedge \vec{Y}\right\} .
$$

If equality holds, then $S$ is invariant under the action of $G$ by 4.11 . If not, then we can construct $T \in \mathrm{I}_{m}\left(\mathbf{R}^{n}\right)$ by 4.9 such that

1. $\partial T=B$,

2. $T$ is invariant under the action of $G$, and

3. $\Psi(T) \leq \Psi\left\{\left[S\left\llcorner\left(Z \wedge Y^{*}\right)\right] \wedge \vec{Y}\right\}<\Psi(S)\right.$,

contradicting the assumption that $S$ is a $\Psi$-minimizing integral current.

\section{REFERENCES}

1. D. Bindschadler, Invariant solutions to the oriented plateau problem of maximum codimension, Trans. Amer. Math. Soc. 261 (1980), 439-461.

2. J. E. Brothers, Invariance of solutions to invariant parametric variational problems, Trans. Amer. Math. Soc. 262 (1980), 159-179.

3. J. Dadok, Polar coordinates induced by actions of compact Lie groups, Trans. Amer. Math. Soc. 288 (1985), 125-137.

4.

5. H. Federer, Geometric measure theory, Springer-Verlag, New York, 1969.

6. S. Helgason, Differential geometry, Lie groups, and symmetric spaces, Academic Press, New York, 1978.

7. J. C. Lander, Area-minimizing integral currents with boundaries invariant under polar actions, Ph.D. Thesis, Massachusetts Institute of Technology, 1984.

8. H. B. Lawson, Jr., The equivariant Plateau problem and interior regularity, Trans. Amer. Math. Soc. 173 (1972), 231-249.

9. F. Morgan, On finiteness of the number of stable minimal hypersurfaces with a fixed boundary, Indiana Univ. Math. J. 35 (1985), 779-833.

10. R. S. Palais and C.-L. Terng, A general theory of canonical forms, preprint.

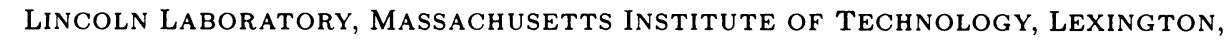
MASSACHUSETTS 02173 